Abstract

\title{
Entropy-Based ECG Biometric Identification +
}

\author{
João M. Carvalho, Susana Brás and Armando J Pinho \\ Institute of Electronics and Informatics Engineering of Aveiro, University of Aveiro, Aveiro, Portugal \\ + Presented at the Entropy 2021: The Scientific Tool of the 21st Century, 5-7 May 2021; Available online: \\ https://sciforum.net/conference/Entropy2021/. \\ Published: 5 May 2021
}

There is a great interest nowadays in using ECG for biometric identification, due to some of its intrinsic properties. There are different proposals in the literature to solve this task. Most of them based on feature extraction and machine learning methods applied on those features and, recently, others based on deep learning algorithms applied directly on the signals-providing better results, but requiring a much higher computational cost. In this study we aim to show how an approach based on compression methods can be used to perform this task. Our approach uses the notion of relative compression to provide a measure of similarity from a new signal to the different participants present on the database. This is an approach which relies heavily on the entropy of the data sources, as the compressors used to perform the relative compression are based on finite-context models (similar to Markov models). We aim to show that it is possible to perform biometric identification using ECG signals using this methods, even without applying any kind of fiducial point detection on the ECG, making the method, in theory, more general than for this specific task or type of signal. Our results show that this approach is feasible in practical terms and provide competitive results, achieving an accuracy of around $89.3 \%$ using an publicly available database with 25 participants.

(C) 2021 by the authors. Licensee MDPI, Basel, Switzerland. This article is an open access article distributed under the terms and conditions of the Creative Commons Attribution (CC BY) license (http://creativecommons.org/licenses/by/4.0/). 